TM-1111

2300.000

\title{
CAMAC Crate Controller \\ Diagnostic Test Procedures
}

James T. Meadows

$$
\text { July 1, } 1981
$$


CAMAC Crate controller

Diagnostic Test Procedures

BY

James T. Meadows

July 1,1981 


\section{CONTENTS}

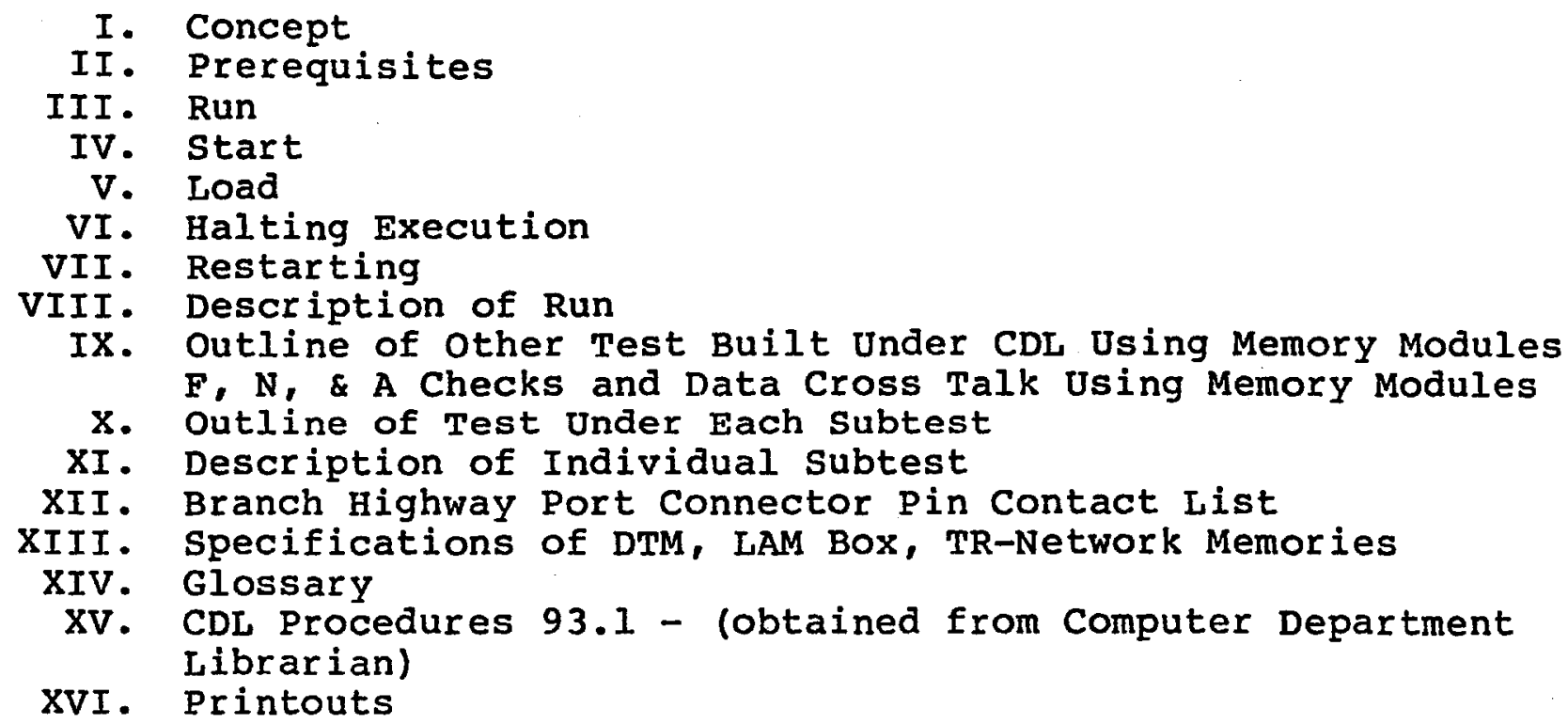




\section{Concepts}

I.I Abstract

The basic idea of this diagnostic routine is to check out a parallel type Al CAMAC crate Controller using "CDL" •

\section{2 Program}

This program was written in "CAMAC Diagnostic Language" (CDL-PN93.1) in a modular design. Modular design allows the technician checking out the crate controller to run individual parts of the whole test. So, when the program detects a failure, it leads the technician to a specific fault area. The technician can now loop on that individual subroutine for further testing.

\section{I.3 Program Synopsis}

The full test runs a quick check (Pretest Run) of the Branch Driver's abilities to properly detect and latch data, to see if any crates are on-line, transfer its register and data buffers to "core" locations, see if a DTM-399 Dataway Display Module is in any crate (at station - "l", specifically) and printout if it saw a module and crate.

Next the test runs a set-up program which allows the technician to enter locations of the test module, the LAM latch module, and memory modules which will be used later in the testing \& checkout of "Data Bits". 
The program then checks to see if any crates can be seen "on-line". If so, the program runs the individual sub-tests, as listed on the printouts and descriptions of the individual "sub-routines".

I.4 The program runs the loop BZ, SETI, SETC, CLRI, EBD, and TSTBD thru 100 cycles. During that time the program lets you see and test the crate controller indicator lights. The "D" (Branch Demand) light is to be cycled by the technician by pressing the lam latch extension box buttons.

I.5 At the end of the 100 cycles, the program sets up to test "GRADED-L" operations using the LAM box or the DTM-399/299 test module. The program prompts the technician on what to do and waits for the "LAM" in the dataway to cause a "Branch Highway" D-demand to occur. The program "thanks you" and suggests a further important operation to proceed.

NOTE: A full graded-I connector is required on the crate controller to assure any module at any slot/station can produce a "LAM", to crate, to "D", to "BD" Cycle under "TSTGL" subroutine. 


\section{Prerequisites}

A. LSI, PDPII with 28K of Memory

B. Floppy, Disk, Tape, etc.

C. DTM 399/299 "Dataway Test Display Module" at

Station 1. See Attached Data Sheet.

D. LAM Latch Module with Extension box any other station (2-23). See Attached Data Sheet.

E. CAMAC Crate with power supply

F. Branch Driver, JY4II or BDOlI

G. 2 TR-Network Memory Modules - See Attached Data Sheet. III. TO Run
A. Enter - R CDLBD CR>
B. Enter - READ 'Crate.CDL/N' CR>
C. Enter - Run (If this is second time through)

NOTE: Program is self-starting otherwise.

IV. starting
A. Run CR>
B. Enter - procedure name of subtest for running individual parts of Diag. See "CDL-PN 93.1".

V. Loading

NOTE: Loading of floppy disk and other media are usually done with a "local" set of instruction.

"Local" - (at the site)

VI. Halting Execution

A. Strike/Push: (CR) twice stops CDL programs

B. Enter "control $c$ " halts CDL and returns to RTII monitor 
VII. Restarting in subroutines

A. Enter - Subroutine name - runs the individual routine as Per PN93.1

B. Enter - subroutine name $x x(x=$ Decimal) - runs routine " $X$ " times then stops 
VIII. Description of "Run"

VIII. 1 Run

a) Sets up to loop 100 times - Default is 100 times

b) Pretest

1. Disables $\mathrm{x}$-fail alarms - Note 1

2. Checks Drivers DATA Registers - 24 Bits

3. Checks Drivers ability to Latch and Hold Data

4. Checks for crates \& DTM's at Station 1

5. Quick checks if data can go to and from Driver to DTM Registers - Note 2

6. Re-enable $x$-fail alarms

7. Call next step

c) Set-up - where is "everything"

d) Again a quick check if any crates are on-line

e) Explain XI \& QL meanings

f) Cycles BZ, SETI, CLRI, EBD, TSTBD, checks "X" \& " $Q "$ - NOTE 3

g) Loop 100 times

h) Test graded-L connector \& crate controllers Graded L operations

i) Re-enable function x-failure in program - CDL - NOTE 4

j) Call to set up For FNA test and more comprehensive data \& function checks

End 
NOTES

1. See Glossary for terminology.

2. DTM must be in slot 1 of crate.

3. Sub-routines

4. See Glossary for some terminology. 
IX. Outline of Other Test

IX.1 CRTRUN.Run

(this runs the test)

1. Pretest

2. Set Up

3. Loop 100 Times

4. TSTGL

End

IX.2 CRTLSI.RUn

(this is a subpart called under

CRTRUN)

1. NOCRAT

2. SETI

3. SETC

4. BZ

5. CLRI

6. EBD

7. TSTBD

IX. 3 CRTLS2.Run

(this is a subpart called under CRTRUN)

1. TSTGL

2. SNR - NOTE: May not be installed due to size limitation of "Procedure Buffer" can be run individually as per PN93.1.

End

IX. 4 FNATS1.CDL

(this is a full F, N, \& A test of the crate controller) 
IX.5 F\&N\&A\&Data Cycles

Checking Crosstalk

End

IX. 6 SKPLSI.CDL

(this is a random block $x$-fer of data in and out of (AMAC)

1. Checks full data patterns and system

2. Checks CAMAC to Driver Data

End

IX. 7 SNR Run

1. Sets up SNR to do operations to DTM and or Memory Modules 
x. Descriptions of Individual Sub-Test Run Procedures

NOCRAT.CDL - (found on Media as NOCRAT.CDL)

\section{X.1 NOCRAT}
a) Set CS 1
Do a Reset
b) Set *x 1
Set crate variable to Crate 1
c) Set *F 0
Set variable function to "0"
d) $\operatorname{set} \star \mathrm{N} 1$
Set station variable to station 1
e) Set ${ }^{B} 1$ Set assumed station of DTM 399 to station "I"
f) Disable X-alarm failure printouts
g) Do a CAMAC cycle FNAC - F 0 l 0 (*x = crate)
h) Check value of "XL": if set a crate \& module is at Station " 1 "
i) increment crate No.
j) Check if crate No. is greater than "8"
k) Re-enable $x$-fail printouts and
End

\section{X.2 CRTLSI.Run}

SETI
a) Enable "I" on crate "FNAC"
F $2630 \quad 9 * C$
b) test " $\mathrm{X}$ " of driver to see if crate \& cycle was accepted
c) test crate controller for $X \& Q$,
d) if "I" is set $(Q=I)$
e) If $x$ not set Say so
If $Q$ not set say so 

f) Print test failed
print value of " $X$ " \& " $Q$ "
g) End

\section{X.3 CLRI}
a) Disable "I"/turn off "FNAC"
F $2430 \quad 0 \quad * C$
b) Check " $\mathrm{X}$ "; accepted by crate
c) Test crate for " $X$ " \& " $Q$ " $Q=1$ if "I" is still set
d) If " $\mathrm{x}$ " not $=1$ say so
e) If " $Q$ " not $=0 \quad$ print failure
End

X. $4 \quad$ SETC

a) Disable $\mathrm{X}$-fail alarms

set "C" "FNAC"

F $26 \quad 28 \quad 9 * C$

b) Wait for $100 \mathrm{milliseconds}$ to complete CAMAC cycle

c) Check if " $\mathrm{X}$ " accepted function

d) Re-enable $x$-fail alarms

End 
X.5 BZ
a) Disable x-fail alarms
b) Do a BZ from "Driver" set CS 1 (cycles BZ Branch Highway Line)
c) Do BZ to crate "FNAC"
F $26 \quad 28 \quad 8 \quad$ *C
d) Check $x=1 \quad$ accepted
e) Check $Q=0 \quad$ okay
End

X. 6 EBD
a) Disable $x$-fail alarms
b) Enable "BD" "FNAC"
F $263010 * C$
c) Check $x=1$
$Q=0$
d) Check if enabled: F, 27, 30, 10, *C $x=1$
e) $Q=1$ (means crate $B D$-enable is set)
f) Re-enable x-fail alarm
End

X.7 TSTBD

a) Enable BD "FNAC"

F, $26,30,10,{ }^{*} \mathrm{C}$ 
b) Check " $\mathrm{X}$ " = 1

c) Check if " $Q$ " not previously set

d) If " $Q$ " previously set then

e) Reset $=$ set $\operatorname{cs} 1$

f) Now check if " $Q$ " not set

$F, 27,30,10,{ }^{*} \mathrm{C}$

g) Check $Q$ not set print failure if set

h) Enable "BD on" FNAC

$\mathrm{F}, 26,30,10,{ }^{\mathrm{C}} \mathrm{C}$

this is to allow the 100 iteration cycle under run to set and light the "D" light on the crate controller

End

X.8 CRTLS2.Run - Crate List No. 2 (found on Media as CRTSL2.Run)

TSTGL
a) set timer
b) This checks crate controller for set \& test of "BD"
c) Enables DTM 399./299 for "LAM" Enable to Dataway.
d) Gives aid and information for "LAMS" from LAM-Latch box and DTM 399/299
e) Print "I" minutes still no "LAM"; if no LAM was received for "I" (one) minute or LAM "lost".
f) Check if driver can see demand from crate controller
g) Do FNAC Graded-L
h) $\mathrm{F}, 0,30,7,{ }^{\star} \mathrm{C}$ 
i) Say Got It

j) Check $X \& Q \quad Q=1$ for Graded $L$

k) Print value of button pushed on box

1) Enable $x$-fail

End 
TABLE VII Contact Assignments at Branch Highway Ports: By Contact Numbers

\begin{tabular}{|c|c|c|c|c|c|c|c|}
\hline $\begin{array}{l}\text { Contact Signal } \\
\text { Sign }\end{array}$ & $\begin{array}{l}\text { Contact Signal } \\
\end{array}$ & $\begin{array}{l}\text { Contact Signal } \\
\text { Signal }\end{array}$ & $\begin{array}{l}\text { Contact Signal } \\
\qquad\end{array}$ & $\begin{array}{l}\text { Contact Signal } \\
\end{array}$ & $\begin{array}{l}\text { Contact } \\
\text { Signal }\end{array}$ & $\begin{array}{l}\text { Contact Signal } \\
\qquad \text { S }\end{array}$ & $\begin{array}{l}\text { Comract } \\
\text { Signal }\end{array}$ \\
\hline $1 \mathrm{BA} \mid(\mathrm{R})$ & $18 \mathrm{BN} 2(\mathrm{R})$ & 35 BCRA & 52 BCR7(A) & $69 \mathrm{BCR} 7$ & $86 \quad B R W 11(R)$ & 103 BRWg & 120 ВTB6 \\
\hline $2 \mathrm{BA} 2(\mathrm{R})$ & $19 \mathrm{BN} 4(\mathrm{R})$ & $36 \mathrm{BN} 1$. & $53 B F 1(f)$ & $70 \mathrm{BF} 1$ & S7 BAW 12(R) & 104 BRW10 & 121 Br87 \\
\hline $3 B A 4(R)$ & $20 B N 8(R)$ & $37 \mathrm{BN} 2$ & $54 \quad B F 2(R)$ & $71 \mathrm{BF} 2$ & $88 B R W 13(R)$ & 105 BRW11 & $122 B T B 7(A)$ \\
\hline $4 B A B(R)$ & $21 \mathrm{BN} 16(\mathrm{~A})$ & 38 BN4 & $55 B F 4(R)$ & $72 \mathrm{BF} 4$ & 89 BRW14(R) & 106 BRW12 & $123 \quad 8 T B 5$ \\
\hline $53 \vee 1(R)$ & 22 ВТВЗ(R) & 39 BN8 & $56 \quad B F 8(R)$ & $73 \mathrm{BF8}$ & 90 BRW15(R) & 107 BRW13 & 124 BRW21 \\
\hline $6 \quad 3 \vee 2(R)$ & $23 \mathrm{BA} 2$ & $408 N 16$ & $57 B F 16(R)$ & 74 BF 16 & $91 \quad B R W 16(R)$ & 108 BRW14 & $125 \mathrm{BRW} 21(\mathrm{~A})$ \\
\hline $7 \mathrm{BV} 3(\mathrm{R})$ & 24 BA4 & $41 B A 1$ & 58 втв3 & $75 \mathrm{BSC}(\mathrm{R})$ & 92 BTBA(A) & 109 BRW15 & 126 BRW22 \\
\hline $8 B \vee 4(R)$ & $25 B A 8$ & $42 B G(R)$ & $59 \quad \mathrm{BG}$ & 76 BRW1(R) & 93 BRW1 & 110 BRW 16 & 127 BRW22(R) \\
\hline y $B \vee 5(R)$ & $26 B V 1$ & $43 B D(R)$ & $60 B D$ & 77 BRW2(R) & 94 BRW2 & $111 \mathrm{BSC}$ & $128 \quad B R W 23$ \\
\hline $10 B T B 1(R)$ & 27 BV2 & $44 \mathrm{BO}(\mathrm{R})$ & 6180 & 78 BRW3(R) & 95 BRW3 & 112 BRW17 & $129 \mathrm{BRW} 23(\mathrm{R})$ \\
\hline 11 BTB2 & 28 BV3 & $45 B Z(R)$ & $62 \mathrm{BZ}$ & 79 BRW4(R) & 96 BRW4 & 113 BAW17(R) & 130 BRW24 \\
\hline 12 BTB2(R) & $298 V 4$ & $46 \mathrm{BTA}(\mathrm{R})$ & 63 BTA & 80 BRW5(R) & 97 BRW5 & 114 BRW18 & 131 BRW24(A) \\
\hline $13 B C R 1(R)$ & $308 \vee 5$ & $47 \mathrm{BV} 6(\mathrm{R})$ & 64 BV6 & 81 BRW6(R) & 98 BRW6 & 115 BRW18(R) & 132 вТВ4 \\
\hline 14 BCR2(R) & 31 BTB1 & $48 \mathrm{BV} 7(\mathrm{R})$ & 65 BV7 & 82 BRW7(R) & 99 BRW7 & 116 BRW19 & \\
\hline 15 BCR3( $\mathrm{K})$ & $32 \mathrm{BCR} 1$ & $498 \times(R)$ & $66 \mathrm{BX}$ & 83 BRWB(R) & 100 BRWB & 117 BRW19(R) & \\
\hline 16 BCA4(R) & 33 BCR2 & $50 \mathrm{BCR5}(\mathrm{R})$ & 67 BCK5 & 84 BRW9(R) & 101 BTBG(R) & 118 BHW20 & \\
\hline $17 \mathrm{BN} 1(\mathrm{R})$ & $34 \quad B C R 3$ & 51 BCR6(R) & 68 BCR6 & $85 B R W 1 O(R)$ & $102 \mathrm{BTBS}(\mathrm{R})$ & 119 BRW2O(R) & \\
\hline
\end{tabular}

\section{POLARIZING BOSS PROJECTIONS ASSOCIATED RETURN CONTACT}

N.B. $B R W 1(R)$ is the return line corresponding to BRW1

SIGNAL CONTACT

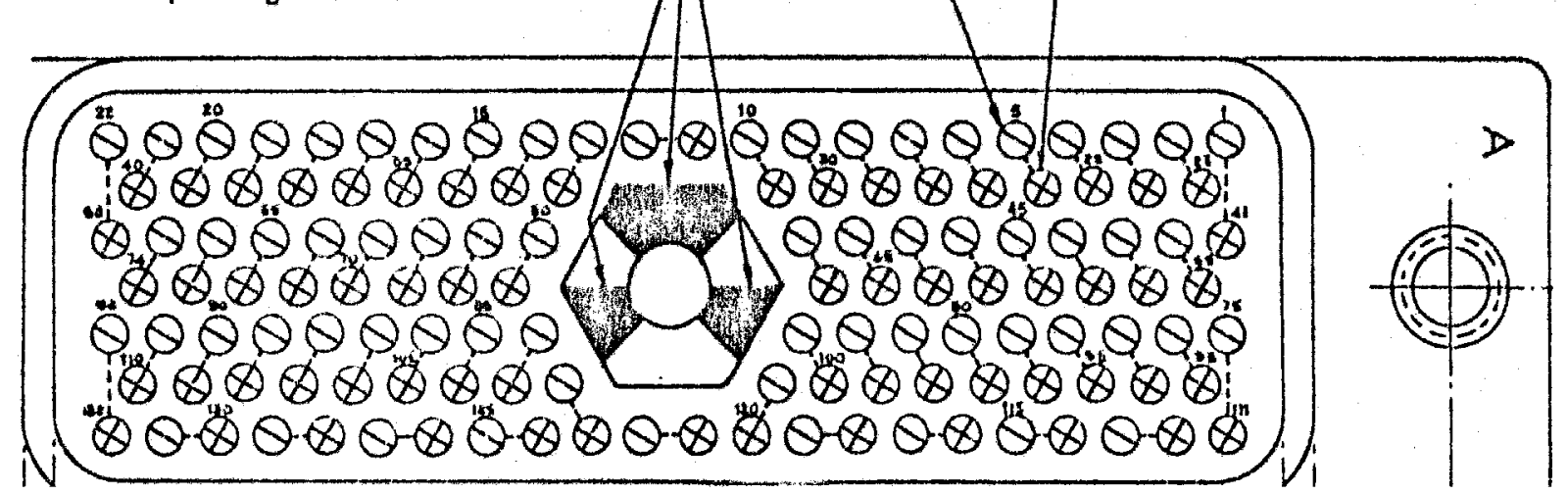




\section{CAMAC TEST MODULES THAT INFORMS PEOPLE AND COMPUTERS WHAT IS REALLY HAPPENING ON THE DATAWAY}

\section{FEATURES:}

MODEL DTM-299 (Standard Version)

-Displays ALL dataway signal

- Both Track and Latch mode

- Manual LAM generation

- Read of previous dataway cycle

Data and Status F, A, etc.

-Stretched display of B, S1, S2, L

MODEL DTM-399 (Enhanced Version)

-Power supply failure detection

-Ambient temperature monitor

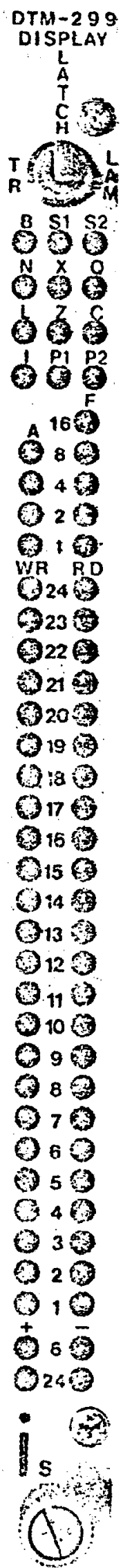

\section{DESCPIPTION:}

This series of Dataway Test Modules is designed for use as a low-cost diagnostic, test and display aid for troubleshooting CAMAC, from both a hardware and software viewpoint. Status and dataway conditions can be seen at a glance, also computer controlled systems can run self diagnostics through to the dataway level. (Confidence factor for system programmers)!

Front panel LED indicators display the status of all dataway signals in either Latch or Track (continuous) mode. Write data, control signals, and status for the previous dataway operations may be read back for system self diagnosis. A front panel manual LAM switch is also available to test the crate and software LAM handling capability.

In addition to these features the MODEL DTM-399 includes monitoring of ambient temperature and the four standard CAMAC power supplies. Should a preset limit be exceeded by high ambient temperature, a voltage failure, current spike or power supply oscillation a LAM will be generated. The detection and logic circuitry is powered by voltage steering and any supply may fail without affecting the modules operation.

\section{PRICE DTM-299 \$325.00 DTM-399 $\$ 425.00$}




\section{COMAMANDS:}

oT:3-299

NA[0]F[0] Read data $Q, X=1$

Reads previously Latched write data onto Dataway

read linds. R1-R24

NA[0]F[1] Read status $Q, X=1$

Reads previousiy Latched status, function code.

subaddress, etc. onto Dataway read lines, plus

reads position status of front panel switch.

\begin{tabular}{|c|c|c|c|c|c|c|c|c|c|c|c|c|c|c|c|}
\hline R17 & 15 & 14 & 13 & 12 & $1 /$ & 10 & 9 & 8 & 7 & $L$ & 5 & 4 & 3 & 2 & R1 \\
\hline TR & 0 & P1 & P2 & C & $Z$ & 1 & F16 & F8 & $F_{4}$ & F2 & $F 1$ & A8 & A4 & $A 2$ & A1 \\
\hline
\end{tabular}

NA[0]F[8] Test LAM $X=1$

Test LAM status, $Q=1$ if set
NA[0]F[10] Reset LAM $\quad 0, X=1$

Resets LAM request.

DTM-399 [ADDITIONAL COMMANDS]

NA[0]F[1] Read Status $0, X=1$

\begin{tabular}{|l|l|l|l|l|l|l|}
\hline M24 & \multicolumn{3}{|c|}{ R18 } \\
\hline LAM & +6 & -6 & +24 & -24 & TEMP & \\
\hline
\end{tabular}

NA[0]F[24] Disable LAM $\quad Q, X=1 \quad$ NA[0]F[26] Enable LAM $\quad 0, X=1$

\section{SPECIFICATIONS:}

SIGNALS DISPLAYED

Latched at S1

Read $R(24) \quad$ Write $W(24)$

Function $F(5) \quad$ Subaddress $A(4)$

Stn. No. N O Response 0

P1 Bus P1 $X$ Response $X$

P2 Bus P2

Latches at $\mathbf{S 2}$

Initialize $\mathrm{Z}$

Stretched [200 MS]

Strobe $1 \quad$ S1

Busy

B

Strobe $2 \quad$ S2

LAM L
Continuous [Track]

\pm 6 Volts $\quad 6(2)$

Inhibit $\quad$ (1)

Power Requirements

$+6 \mathrm{~V} \cdot 18 \mathrm{~A}$ Max

$-6 \mathrm{~V} . \pm 24 \mathrm{~V}-16 \mathrm{ma}$.

Operational Modes

LATCH - Dataway signals are latched with the appropriate strobe

TRACK - Real Time continuous display of dataway signals

Mechanical

Single width module

\section{DTM-399 [ONLY]}

Temperature set point range: $25-60^{\circ} \mathrm{C}$

Voltage sel point range:

$+6=+3$ to $+6 \mathrm{v}+24=+21$ to $+24 \mathrm{v}$

$-6=-3$ to $-6 \mathrm{v} \quad-24=-21$ to $-24 \mathrm{v}$

Minimum voltage excursion time for detection (vollage spike) $=1$ usec

Reference stability $= \pm .1 \% 24$ hours. 
COMMANDS

NA[0]F[0] Read Data $Q, X=1$

Must be in the Latch mode. Reads latched write data

from previous dataway operation onto corresponding

dataway. Read Iines 1-24.

\section{NA[0]F[1] Read Status}

Must be in the Latch mode. Reads latched status,

function code and sub-address from previous data-

way operation.

\begin{tabular}{|l|l|l|l|l|l|l|l|l|l|l|l|l|l|l|l|l|l|l|l|}
$\mathrm{R} 17$ & 11 & 15 & if & 13 & 12 & 11 & 10 & 9 & 8 & 7 & 6 & 5 & 4 & 3 & 2 & $\mathrm{R} \phi$ \\
\hline $\mathrm{TR}$ & $\mathrm{Q}$ & $\mathrm{X}$ & $\mathrm{P} 1$ & $\mathrm{P} 2$ & $\mathrm{C}$ & $\mathrm{Z}$ & $\mathrm{T}$ & $\mathrm{F} 16$ & $\mathrm{~F} 8$ & $\mathrm{~F} 4$ & $\mathrm{~F} 2$ & $\mathrm{~F} 1$ & & & & $\mathrm{~A} 8$ & $\mathrm{~A} 4$ & $\mathrm{~A} 2$ & $\mathrm{~A} 1$ \\
\hline
\end{tabular}

R10, [I], RI7 and [TR] may be read in either Track

or Latch mode. Track is an internal signal. When

active, indicates select switch in Track mode.

NA[0]F[8]

$x=1$

Test LAM status, $Q=1$ if set.

NA[0]E[10] Reset LAM

$Q, X=1$

Resets manual LAM request.

\begin{tabular}{|c|c|c|c|c|c|c|}
$\mathrm{R} 24$ & 23 & 2 & 2 & 20 & 1 & $\mathrm{R} 18$ \\
\hline $\mathrm{MAN}$ & & & & & & \\
$\mathrm{LAM}$ & +6 & -6 & +24 & -24 & TEMP & - \\
\hline
\end{tabular}

NA[0]F[24] Disable LAM

$Q, X=1$

NA[0]F[26] Enable IAM

$Q, X=1$ 


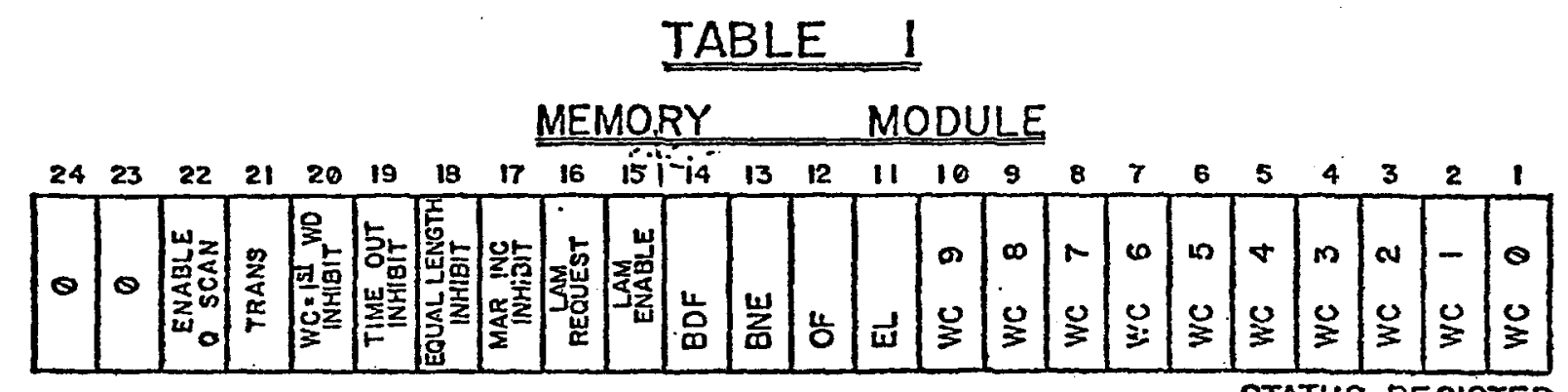

STATUS REGISTER

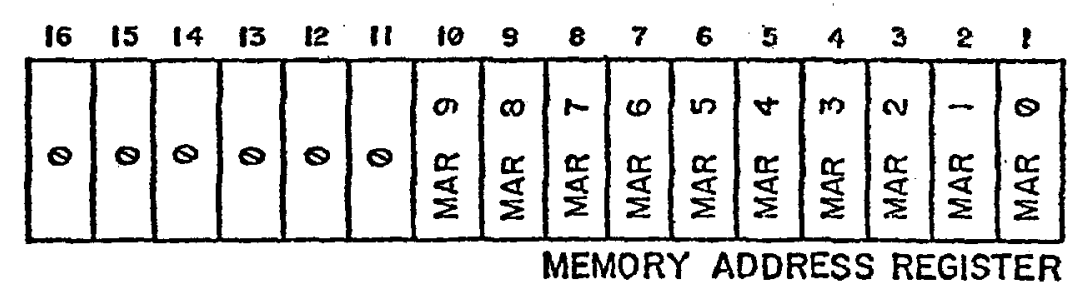

\section{CAMAC COMIMANDS}

$F(0) \cdot A(n)$

$F(1) \cdot A(O)$

$F(1) \cdot A(1)$

$F(1) \cdot A(2)$

$F(2) \cdot A(n)$

$F(8) \cdot A(0) \cdot S 1$

$F(10) \cdot A(0) \cdot S 1$

$F(11) \cdot A(0) \cdot S I$

$F(I 1) \cdot A(2) \cdot S I$

$F(16) \cdot A(n) \cdot S !$

$F(17) \cdot A(1) \cdot S 1$

$F(17) \cdot A(2) \cdot S I$

$F(18) \cdot A(n) \cdot S 1$

$F(19) \cdot A(0) \cdot S i$

$F(24) \cdot A(0) \cdot S I$

$F(26) \cdot A(0) \cdot S i$

$F(27) \cdot A(0) \cdot S t$

$(z+c) \cdot s 2$

\section{FUNCTION}

MEMORY BLOCK READ

READ WC \& STATUS REGISTER

READ SINGLE MEMORY LOCATION [AS f(MAR)]

READ MAR

OPTIONAL BLOCK READ [INSTEAD OF F(O)]

TEST LAM $Q=1$ IF LAM REQ. - MASSK

RESET LAM REQ.

CLEAR WC \& STATUS REGISTER

CLEAR MAR

MEMORY BLOCK WRITE

LOAD SINGLE MEMORY LOCATION [AS f(MAR)]

LOAD MAR

OPTIONAL BLOCK WRITE [INSTEAD OF F(16)]

SELECTIVE SET WC \& STATUS REGISTER

DISABLE LAM

ENABLE LAM

TEST LAM $Q=1$ IF LAM REQ.

CLEAR ALL REGISTERS

NOTE: " $X$ " RESPONSE GENERATED FOR ALL VALID COMMANDS AND

" $Q$ " RESPONSE GENERATED FOR READ AND YIRITE OPERATIONS 


\section{Glossary}

Abort

BDO11/JY411

BIT

BIT Positions

Binary

Boot

Branch Driver

Branch

Branch Highway

Buffer

CDI

CORE

Demand

Dataway
Quit operations in progress either by normal expiration or failure to complete properly. CAMAC drivers designed for DEC PDP-11

Systems

Either on or off at a specific BIT position

Bits from $(1-24)$ for a CAMAC

word 0-15 for a computer word.

A numerical weighting system used in

computers

Load system computer with a program JY411, BDO11.

A junction in a program where a decision is made to jump to another portion of the program.

Communication lines between crate controller and branch driver.

A block of computer memory used for storage of a lot of computer words. CAMAC Diagnostic Language - PN-93.1.

A computer memory location.

A CAMAC system request from a crate controller

The back plane pin contacts inside a CAMAC crate at each station. 
FNAC

Floppy disk

DEC RK05

LAM

Graded-I

Loop

Local

OCTAL

Procedure

RT-11

$x$-fail
Function, station, sub-address, crate A flexible magnetic disk used as

a mass storage device with computers (holds programs \& data)

Same as above but is rigid and holds

more data

CAMAC "Dataway" request "Look at Me"

(initiates crate controller "Demand")

A crate controller operation that

places the dataway stations "LAM lines" on to

the BRW lines (crate controller needs a

graded-L connector on the back panel)

A repeating set of instruction or

"procedures" inside a program. (See

procedures)

Wherever a test or computer system is at.

${ }^{10} 10=10$ Decimal

$10_{8}=8$ octal

$10_{2}=2$ Binary

Example: Binary $111011011 \quad 001_{2}$

$\begin{array}{lllll}\text { octal } & 7 & 3 & 3 & 1_{8}\end{array}$

A single or group of instructions

under CDL starting with "Define" and ending with "END" .

Is the operating system that CDL

is controlled and run under.

A printout that can occur when No-X 
(improper CAMAC function) is detected by CDI'S monitoring and error checking section. The printout can occur when a non-existent crate is called or an invalid function is attempted to a module that is not there or cannot be done by that module.

$\mathrm{X}$-fail is valid for BDoll's only if a crate is not available.

$\mathrm{BRW}-(\mathrm{XX})$

"BX"

"BQ"

"BD"

"GL"

"BN"

"BF"

"BA"
Branch Highway Read and Write Iines

CAMAC function accepted

Used as a condition indicator in CAMAC

modules \& crate controllers

Branch Demand

Graded-L

Branch N-line

Branch F-line

Branch A-line 
XVI. Printouts

XVI.I How to Obtain Copies

XVI.2 Printed on Console

1. Under RTII Enter - R PIP (CR)

2.

Enter - TT: <CRATE.CDL (CR)

3. or Enter - TT: <CDL Program Name.Ext (CR)

XVI. 3 Printed on Line Printer

1. Under RTll Enter - R PIP (CR)

2 .

Enter - LP: $<C D L$ Program Name. Ext (CR) 